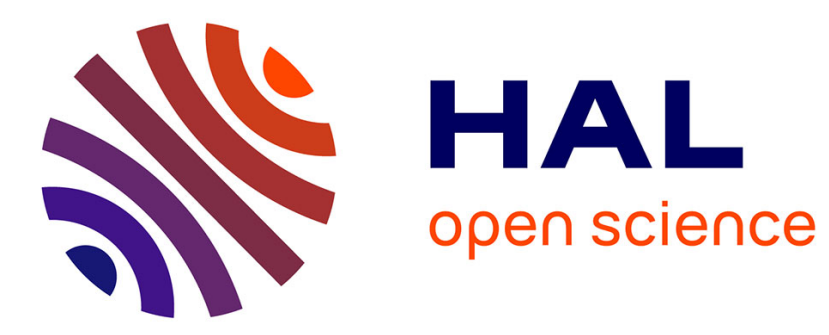

\title{
Spatiotemporal Nonlinear Optical Self-Similarity in Three Dimensions
}

\author{
S. Chen, J.M. Dudley
}

\section{To cite this version:}

S. Chen, J.M. Dudley. Spatiotemporal Nonlinear Optical Self-Similarity in Three Dimensions. Physical Review Letters, 2009, 102, pp.233903. 10.1103/PhysRevLett.102.233903 hal-00453028

\section{HAL Id: hal-00453028 \\ https://hal.science/hal-00453028}

Submitted on 3 May 2021

HAL is a multi-disciplinary open access archive for the deposit and dissemination of scientific research documents, whether they are published or not. The documents may come from teaching and research institutions in France or abroad, or from public or private research centers.
L'archive ouverte pluridisciplinaire HAL, est destinée au dépôt et à la diffusion de documents scientifiques de niveau recherche, publiés ou non, émanant des établissements d'enseignement et de recherche français ou étrangers, des laboratoires publics ou privés. 


\title{
Spatiotemporal Nonlinear Optical Self-Similarity in Three Dimensions
}

\author{
Shihua Chen ${ }^{1}$ and John M. Dudley ${ }^{2, *}$ \\ ${ }^{1}$ Department of Physics, Southeast University, Nanjing 210096, China \\ ${ }^{2}$ Département d'Optique P. M. Duffieux, Institut FEMTO-ST, UMR 6174 CNRS-Université de Franche-Comté, \\ 25030 Besançon, France
}

(Received 24 November 2008; revised manuscript received 17 May 2009; published 10 June 2009)

We introduce spatiotemporally expanding self-similar light bullets and vortex torus solutions to the $(3+1) D$ nonlinear Schrödinger equation with gain. In the absence of an initial vorticity, we demonstrate an expanding solution with a parabolic intensity profile and linear spatiotemporal chirp. With a nonzero initial vorticity, expanding vortex torus solutions with a centrally embedded phase singularity are found. Such expanding self-similar structures suggest a route towards a new regime of collapse-free spatiotemporal nonlinear optics.

Spatiotemporal localization is a subject of broad interest in physics, spanning such diverse fields as plasma physics, hydrodynamics, and Bose-Einstein condensates. Important studies have also been reported in nonlinear optics, with theory and experiment investigating spatiotemporal effects in many different settings, and for both self-focusing and defocusing nonlinearities [1-6]. Of particular interest in this context are the properties of nondispersing and nondiffracting light bullets [1-3] and the formation of spatiotemporal optical vortex solitons where the wave intensity vanishes at a phase singularity [4-6]. These studies have led to improved insights into beam collapse and filamentation, with important practical consequences for high power nonlinear optics.

In general, the existence of localized structures in optics requires a careful balance among dispersion, diffraction, and nonlinearity, and solutions are often sensitive to beam instabilities. In this regard, however, research in guided wave optics has recently identified nonlinear self-similar propagation as a robust means of avoiding beam or pulse breakup at high power [7-10]. Such an evolution is associated with a characteristic beam or pulse profile that retains the same shape during propagation, even though its amplitude and width scale self-similarly. A limited number of self-similar nonlinear waves (or "similaritons") have been studied in nonlinear optics. Initial research reported linearly chirped parabolic pulses that are selfsimilar asymptotic solutions to the one-dimensional (1D) nonlinear Schrödinger equation (NLSE) with gain [7-9], while other work considered solitonlike similariton pulses in inhomogeneous media where the NLSE has varying coefficients [10]. Related studies in higher dimensions include self-similar beam collapse [11,12], twodimensional beam propagation [13], and solitonlike similariton solutions in graded index media [14].

In this Letter, we consider general self-similar spatiotemporal solutions of the $(3+1) \mathrm{D}$ NLSE with gain. We report a new class of self-similar solutions corresponding to spatiotemporally expanding light bullets and vortex tori and describe analytical predictions that are in very good agreement with numerical simulations. Simulations are also performed in order to show that these self-similar solutions are nonlinear attractors towards which arbitrarily shaped input pulses converge asymptotically. The solutions are obtained in materials with self-defocusing nonlinearity and anomalous dispersion and may suggest a route to the study of high power spatiotemporal dynamics in a regime free of collapse and filamentation.

We express the $(3+1) \mathrm{D}$ NLSE with gain as [1]

$$
i \psi_{z}=-\frac{1}{2 k} \nabla_{\perp}^{2} \psi+\frac{\beta_{2}}{2} \psi_{\tau \tau}-\frac{k n_{2}}{n_{0}}|\psi|^{2} \psi+i \frac{g(z)}{2} \psi,
$$

where $\psi(z, x, y, \tau)$ is the spatiotemporal field envelope propagating along $z, \tau$ is comoving time, $\nabla_{\perp}^{2}$ is the transverse Laplacian acting on $(x, y), \beta_{2}$ is the group-velocity dispersion, $k n_{2} / n_{0}$ accounts for the Kerr nonlinearity, and $g(z)$ is the distributed gain. The wave number in the medium is $k=(2 \pi / \lambda) n_{0}$, and $n_{0}$ and $n_{2}$ are the linear and nonlinear refractive indices, respectively. We consider defocusing nonlinearity $\left(n_{2}<0\right)$ and anomalous dispersion $\left(\beta_{2}<0\right)$, a combination accessible in novel (e.g., quadratic) nonlinear materials $[15,16]$. We define normalized variables $Z=z / L_{\mathrm{df}}, X=x / w_{0}, Y=y / w_{0}$, $T=\tau /\left(L_{\mathrm{df}}\left|\beta_{2}\right|\right)^{1 / 2}, \quad G(Z)=g L_{\mathrm{df}}, \quad$ and $\quad \Psi=$ $\left(k\left|n_{2}\right| L_{\mathrm{df}} / n_{0}\right)^{1 / 2} \psi$. The resultant normalized propagation equation is

$$
i \Psi_{Z}=-\frac{1}{2} \nabla^{2} \Psi+|\Psi|^{2} \Psi+i \frac{G(Z)}{2} \Psi .
$$

Here $\nabla^{2}=\partial^{2} / \partial X^{2}+\partial^{2} / \partial Y^{2}+\partial^{2} / \partial T^{2}$ is the spatiotemporal Laplacian, and $w_{0}$ is a characteristic transverse width such that $L_{\mathrm{df}}=k w_{0}^{2}$ is the diffraction length.

To find self-similar solutions of Eq. (2), we first write the field in spherical coordinates as $\Psi(Z, R, \theta, \varphi)=$ $\sqrt{E(Z)} U(Z, R, \theta) \exp (i S \varphi)$. Here $R \equiv\left(X^{2}+Y^{2}+T^{2}\right)^{1 / 2}$ is the spatiotemporal radius, $\varphi$ is the azimuthal angle in 
the transverse plane $(X, Y), \cos \theta=T / R$, and $S$ allows for possible integer vortex charge or spin. $E(Z)=$ $E_{0} \exp \left[\int_{0}^{Z} G(\zeta) d \zeta\right]$ is the normalized energy variable, which relates to the energy in real physical units through a multiplication factor $n_{0} \sqrt{L_{\mathrm{df}}\left|\beta_{2}\right|} / k^{2}\left|n_{2}\right|$. With these definitions, $U(Z, R, \theta)$ can be found to obey

$$
\begin{aligned}
2 i U_{Z}+\frac{1}{R^{2}} \frac{\partial}{\partial R}\left(R^{2} \frac{\partial U}{\partial R}\right) & +\frac{1}{R^{2} \sin \theta} \frac{\partial}{\partial \theta}\left(\sin \theta \frac{\partial U}{\partial \theta}\right) \\
& -\frac{S^{2} U}{R^{2} \sin ^{2} \theta}-2 E(Z)|U|^{2} U=0,
\end{aligned}
$$

supplemented by boundary conditions that $U$ must decay exponentially at $R \rightarrow \infty$ and vanish as $(R \sin \theta)^{S}$ at $R \rightarrow 0$. Also, $U$ must satisfy the energy condition $2 \pi \int_{0}^{\infty} R^{2} d R \int_{0}^{\pi} \sin \theta|U(Z, R, \theta)|^{2} d \theta=1$.

We now use a generalized spatiotemporal ansatz for $U$ :

$$
U(Z, R, \theta)=\frac{A(\vartheta) F(\alpha)}{\sqrt{W^{3}(Z)}} \exp [i \Phi(Z, R)],
$$

where $A$ and $F$ are real functions of the self-similarity variable $\vartheta=R / W(Z)$ and $\alpha=\sin \theta$, respectively. The parameter $W(Z)$ is the width, and the phase is assumed as $\Phi(Z, R)=\phi(Z)+i c(Z) R^{2}$, with the parameters $c(Z)$ and $\phi(Z)$ to be determined. Inserting this ansatz into Eq. (3) and equating the real and imaginary parts to zero, we obtain $c(Z)=(1 / 2 W) d W / d Z$ and, exactly,

$$
\begin{aligned}
A^{2} F^{2}= & \eta-\mu \vartheta^{2}+\nu\left[\frac{1}{2 A} \frac{d^{2} A}{d \vartheta^{2}}+\frac{1}{\vartheta A} \frac{d A}{d \vartheta}-\frac{S(S+1)}{2 \vartheta^{2}}\right] \\
& +\frac{\nu}{2 \vartheta^{2}}\left[S-\frac{\alpha}{F} \frac{d F}{d \alpha}\right. \\
& \left.+\left(1-\alpha^{2}\right)\left(\frac{1}{F} \frac{d^{2} F}{d \alpha^{2}}+\frac{1}{\alpha F} \frac{d F}{d \alpha}-\frac{S^{2}}{\alpha^{2}}\right)\right]
\end{aligned}
$$

where

$$
\eta=-\frac{W^{3}}{E} \frac{d \phi}{d Z}, \quad \mu=\frac{W^{4}}{2 E} \frac{d^{2} W}{d Z^{2}}, \quad \nu=\frac{W}{E} .
$$

Note that Eq. (5) exhibits nontrivial asymptotic properties in the limit $Z \rightarrow \infty$ (excluding the trivial solution $A=$ $0)$. For the spinless case $S=0$, the exponential increase in energy due to gain means that $\nu \rightarrow 0$ as $Z \rightarrow \infty$. Thus the last two terms $\propto \nu$ on the right-hand side of Eq. (5) vanish. The solution can thus be determined by the first two terms in Eq. (5), with the function $F$ a constant independent of the angular function $\alpha$. This is also the case for the nonzero spin case $S \neq 0$ when $\vartheta$ and $\alpha$ are comparable to $\nu$. However, the nonzero spin case exhibits more complicated asymptotic behavior at $\vartheta \rightarrow 0$. Specifically, in the vicinity of $\vartheta=0$, the terms related to $\nu$ become dominant and the asymptotic behavior is given by $A(\vartheta) \propto \vartheta^{S}$ and $F(\alpha)=$ $\alpha^{S}$. Note that this is consistent with the above boundary condition at $R \rightarrow 0$ and the results for the case $S=1$ in Ref. [17].
With these asymptotic properties established, we use standard variational methods as in Refs. [4,17] to obtain uncoupled equations for $A$ and $F$ :

$$
\begin{gathered}
A^{2}=\eta-\mu \vartheta^{2}+\nu\left[\frac{1}{2 A} \frac{d^{2} A}{d \vartheta^{2}}+\frac{1}{\vartheta A} \frac{d A}{d \vartheta}-\frac{S(S+1)}{2 \vartheta^{2}}\right], \\
F^{2}=1+\frac{35 \mu \nu}{8 \eta^{2}}\left(\frac{1}{F} \frac{d^{2} F}{d \alpha^{2}}+\frac{1}{\alpha F} \frac{d F}{d \alpha}-\frac{S^{2}}{\alpha^{2}}\right) .
\end{gathered}
$$

It is clear that Eqs. (7) and (8) are (to first order in $\nu$ ) equivalent to Eq. (5) in the asymptotic limit. We now use these two equations to determine both the functional form and the asymptotic scaling relations of the self-similar solutions [7-10].

We begin with the vorticity-free case $S=0$. In terms of Eqs. (7) and (8) and subject to boundary and energy conditions, we can write the asymptotic solution as $A(\vartheta)=$ $\sqrt{15\left(1-\vartheta^{2}\right) / 8 \pi}(\vartheta \leq 1)$ and $F(\alpha)=1$, with $A(\vartheta)=0$ for $\vartheta>1$. This leads naturally to an exact asymptotic selfsimilar solution [8] for the amplitude

$|\Psi(Z, R, \theta, \varphi)|=\sqrt{\frac{15 E(Z)}{8 \pi W^{3}(Z)}}\left[1-\frac{R^{2}}{W(Z)^{2}}\right]^{1 / 2}, \quad R \leq W(Z)$,

with $|\Psi(Z, R, \theta, \varphi)|=0$ for $R>W(Z)$. The pulse width $W(Z)$ and phase offset $\phi(Z)$ are determined by the parametric relations

$$
\begin{gathered}
W^{4}(Z) \frac{d^{2} W(Z)}{d Z^{2}}=\frac{15}{4 \pi} E(Z), \\
\phi(Z)=\phi_{0}-\frac{15}{8 \pi} \int_{0}^{Z} \frac{E(\zeta)}{W^{3}(\zeta)} d \zeta,
\end{gathered}
$$

where $\phi_{0}$ is an initial phase offset. The solution given by Eqs. (9)-(11) describes a localized spatiotemporal pulse with compact support: The pulse has a parabolic intensity distribution and quadratic phase in three dimensions. These characteristics generalize the previously observed parabolic similariton pulses seen in other systems [7-10,13,14].

In what follows, we present specific results for the case of constant gain $G(Z)=G_{0}$. Here the energy and width evolution are $E(Z)=E_{0} \exp \left(G_{0} Z\right)$ and $W(Z)=$ $\left(375 E_{0} / 4 \pi G_{0}^{2}\right)^{1 / 5} \exp \left(\frac{1}{5} G_{0} Z\right)$, respectively [allowing us to easily see the corresponding exponential decrease in $\nu \propto$ $\exp \left(-\frac{4}{5} G_{0} Z\right)$ as discussed above]. The chirp parameter is given by $c(Z)=G_{0} / 10$, with $\Omega_{i}=-\partial \Phi / \partial R_{i}=$ $-G_{0} R_{i} / 5\left(R_{i}=X, Y, T\right)$ denoting the chirp along three axes. The scaling relations for arbitrary gain $G(Z)$ are obtained similarly [10] but are not presented here for brevity.

These expected analytical properties have been confirmed by direct numerical integration of Eq. (2). We use a 3D split-step Fourier technique and consider an ini- 
tially unchirped Gaussian pulse of normalized energy parameter $E_{0}=1.97$ propagating in a medium with gain $G_{0}=6$. These parameters correspond to input pulses of millimeter-scale diameter and microjoule energy and a gain of $\sim 8 \mathrm{~dB}$ over a diffraction length. Figures 1(a) and 1(b) show the simulated transverse phase and amplitude distributions at $Z=2$. The quadratic phase structure in (a) and the compact parabolic shape in (b) are particularly apparent. Figure 1(c) compares the numerical simulations with our analytical results, showing excellent agreement. We stress that, although the pulse expands in both spatial and temporal dimensions, it nonetheless remains spatiotemporally bound, representing a new class of expanding light bullet. The spatiotemporal localization is shown explicitly in the isosurface inset plot.

Additional simulations over a wider parameter range show that the spatiotemporal similariton is a nonlinear attractor into which arbitrarily shaped input pulses converge asymptotically for a given input energy $E_{0}$. To this end, we show in Fig. 2 the evolution trajectories of different initial spatiotemporal pulses [9]. It is clear that, with fixed input energy, the different trajectories (see figure legend) are attracted to the asymptotic sink. Here the phase-space variables are, respectively, calculated from the ratio of the evolving rms spatiotemporal and spectral widths $\Delta R$ and $\Delta \omega$, respectively, relative to those of the expected asymptotic solution at the same distance, given by $\Delta R_{a}=(\sqrt{21} / 7) W(Z)$ and $\Delta \omega_{a}=(\sqrt{21} / 35) G_{0} W(Z)$, respectively.

In the more general case with a vorticity $(S \neq 0)$, Eqs. (7) and (8) admit expanding vortex torus solutions with a zero amplitude and undefined phase at $R \sin \theta=0$. Although these vortex tori exhibit a more complex evolution dynamics $[1,5,6]$, analytic self-similar scaling laws

(a)

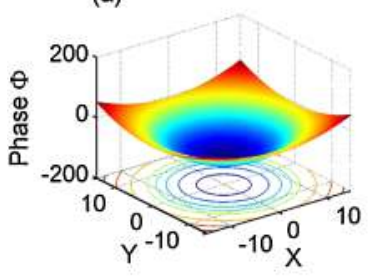

(b)

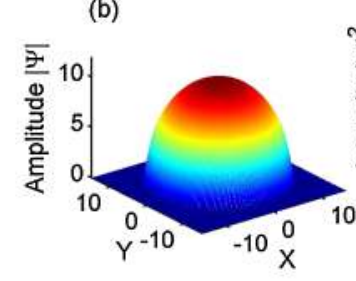

(c)

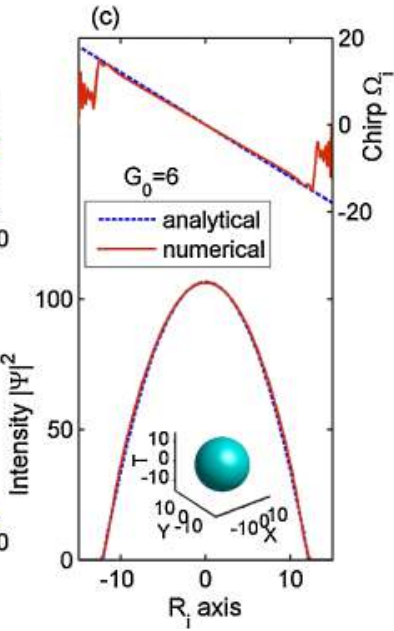

FIG. 1 (color online). Expanding self-similar light bullet at $Z=2$ for an input Gaussian pulse $\Psi(0, R, \theta, \varphi)=\exp \left(-R^{2}\right)$. Parameters are given in the text. (a),(b) Transverse phase and amplitude distributions. (c) Simulated intensity and chirp (solid line) compared with analytical predictions (dashed line). The inset in (c) shows the isosurface plot at $Z=2$. can also be found, both in the neighborhood of the central singularity and at the expanding outer boundary. Specifically, near the phase singularity, the asymptotic behavior of the amplitude obeys $|\Psi| \propto[R \sin \theta / W(Z)]^{S}$, whereas at the expanding outer boundary, it has an asymptotic parabolic shape given by Eq. (9) accompanied by a helical quadratic phase given by $\Phi^{\prime}=S \varphi+\phi(Z)+$ $c(Z) R^{2}$. The scaling relations for the parameters $W(Z)$, $c(Z)$, and $\phi(Z)$ are identical to the vorticity-free case except, of course, at the singularity.

Figure 3 shows simulation results studying the evolution of a Laguerre-Gaussian (LG) input field with a spin of $S=$ 2. Apart from the form of the input field, other parameters are identical as in Fig. 1. Figures 3(a) and 3(b) illustrate the phase and amplitude characteristics at a distance of $Z=2$, clearly indicating the singularity in the quadratic wave front that is helically anticlockwise with a phase increment of $2 \pi S$ for one round trip.

The comparison between the simulations and analytical predictions for this case is shown in Fig. 3(c). This highlights a significant feature of the evolution for the expanding torus. Specifically, although the chirp from simulations is in excellent agreement with the analytic results over the full extent of the torus (excluding, of course, at the singularity), the simulated intensity profile shows significantly better agreement with its analytical prediction in the wings (i.e., $|X|,|Y| \geqslant 10$ ) than closer to the core. This arises from the fact that the self-similar torus evolution is associated with the closing of the central hole and the redistribution of the field energy to approach the asymptotic parabolic form of the outer boundary. This is shown in the isosurface plots in Fig. 3(d) and represents a unique feature of the self-similar evolution of this class of torus structure, in sharp contrast to that of conventional ring tori in other systems $[5,6]$.

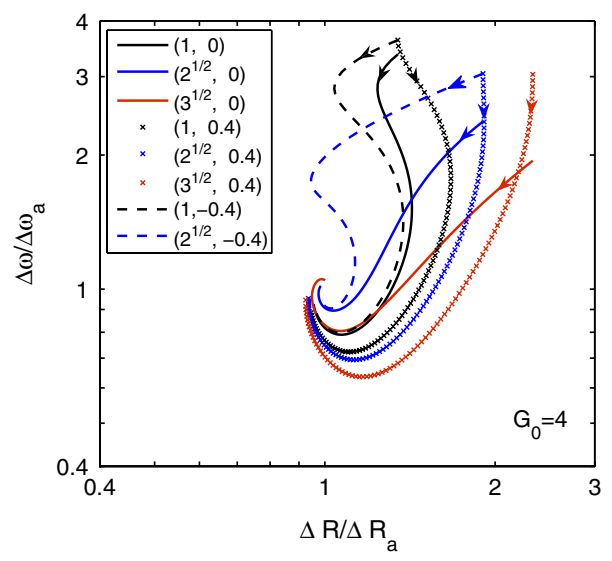

FIG. 2 (color online). Phase-space portrait (log-log scale) showing evolution trajectories towards the expected asymptotic solution for different initial spatiotemporal pulses. Pulses are defined by $\Psi(0, R, \theta, \varphi)=0.5 \sigma^{-3 / 2} \exp \left(-R^{2} / \sigma^{2}+i c_{0} R^{2}\right)$ with parameter sets $\left(\sigma, c_{0}\right)$ given in the legend. Gain $G_{0}=4$, and evolution is followed over 3.6 diffraction lengths. 
(a)
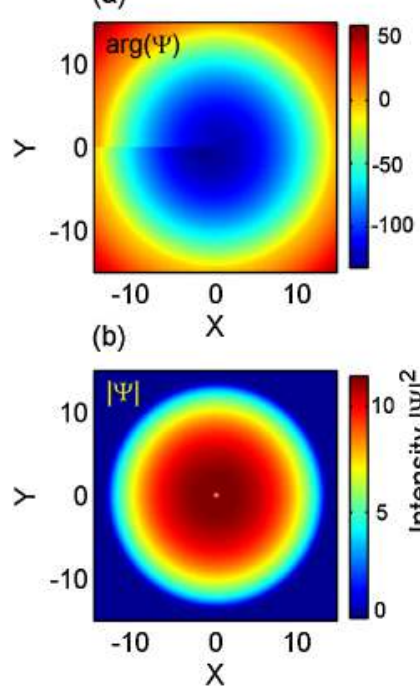

(d) Isosurfaces for $|\Psi|=0.2$

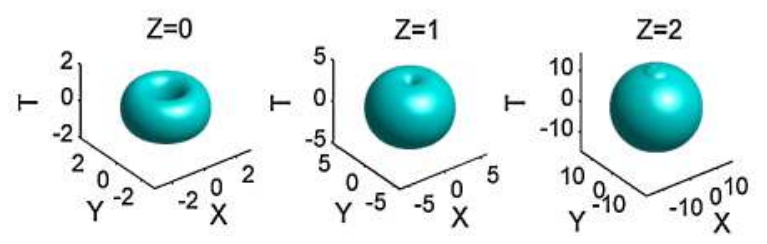

FIG. 3 (color online). Expanding self-similar torus with spin $S=2$ formed from an input LG pulse $\Psi(0, R, \theta, \varphi)=$ $\frac{1}{2} \rho^{2} \exp (i 2 \varphi) \exp \left(-\frac{1}{2} R^{2}\right)$. Other parameters are as in Fig. 1 . (a),(b) Transverse phase and amplitude distributions. (c) Simulated intensity profile and chirp (solid line) compared with analytical predictions (dashed line). (d) Isosurface plots showing the closing of the central hole from $Z=0$ to $Z=2$.

As with the spinless case $S=0$ above, we have used simulations to check that the expanding vortex tori can self-trap from an arbitrary pulse input. A more complicated issue, however, is whether spatiotemporally expanding similaritons are stable against azimuthal symmetrybreaking perturbations. In particular, it has been suggested that azimuthal instability is inevitable for many higherdimensional localized nonlinear entities [4-6,12], and it is natural to consider to what degree the strongly attractive nature of the self-similar solution can resist sensitivity to azimuthal perturbations. Although a complete resolution of this question is beyond the scope of this Letter, preliminary simulations confirm that the expanding similaritons for $S=0$ and $S=1$ indeed resist azimuthal instabilities. A full investigation for higher spins is the subject of ongoing work.

There are several major conclusions to be drawn here. First, we have introduced new concepts in spatiotemporal nonlinear optics in the form of expanding self-similar light bullets and vortex tori. These structures have been studied using both self-similarity analysis and numerical simulations based on the $(3+1)$ D NLSE with gain. In the absence of an initial vorticity, we demonstrate an expanding solution with a parabolic intensity profile and linear spatiotemporal chirp. With a nonzero initial vorticity, expanding torus solutions with a centrally embedded phase singularity are found. Second, the attractive nature of these similariton solutions suggests that they are significantly more robust than conventional spatiotemporal solitons or light bullets whose existence requires more stringent parametric conditions [18]. This may facilitate their experimental observation and may point to possible means to perform bulk amplification of ultrashort pulses without detrimental filamentation effects. In a wider context, these results may also motivate the search for similar higherdimensional self-similar behavior in other systems such as Bose-Einstein condensates where the evolution dynamics is also governed by NLSE-type equations.

S. C. appreciates support from the National Science Foundation of China through Grant No. 10874024. J. M. D. acknowledges support from the Institut Universitaire de France and the French Agence Nationale de la Recherche through Contract No. ANR-08-SYSC-019.

*john.dudley@univ-fcomte.fr

[1] Yu. S. Kivshar and G. P. Agrawal, Optical Solitons: From Fibers to Photonic Crystals (Academic, San Diego, 2003).

[2] Y. Silberberg, Opt. Lett. 15, 1282 (1990); B. A. Malomed et al., J. Opt. B 7, R53 (2005).

[3] X. Liu, L. J. Qian, and F. W. Wise, Phys. Rev. Lett. 82, 4631 (1999); X. Liu, K. Beckwitt, and F. Wise, Phys. Rev. Lett. 85, 1871 (2000); I. N. Towers, B. A. Malomed, and F. W. Wise, Phys. Rev. Lett. 90, 123902 (2003).

[4] A. S. Desyatnikov, A. A. Sukhorukov, and Yu. S. Kivshar, Phys. Rev. Lett. 95, 203904 (2005); A. S. Desyatnikov, Yu. S. Kivshar, and L. Torner, in Progress in Optics, edited by E. Wolf (Elsevier, Amsterdam, 2005), Vol. 47, pp. 291391.

[5] D. Mihalache et al., Phys. Rev. Lett. 88, 073902 (2002).

[6] D. Mihalache et al., Phys. Rev. Lett. 97, 073904 (2006).

[7] D. Anderson et al., J. Opt. Soc. Am. B 10, 1185 (1993).

[8] M.E. Fermann et al., Phys. Rev. Lett. 84, 6010 (2000); F. Ö. Ilday et al., Phys. Rev. Lett. 92, 213902 (2004).

[9] J. M. Dudley et al., Nature Phys. 3, 597 (2007).

[10] V. I. Kruglov, A. C. Peacock, and J. D. Harvey, Phys. Rev. Lett. 90, 113902 (2003); S. Chen et al., Phys. Rev. E 72, 016622 (2005); S. A. Ponomarenk and G.P. Agrawal, Phys. Rev. Lett. 97, 013901 (2006); J. Belmonte-Beitia et al., Phys. Rev. Lett. 100, 164102 (2008).

[11] K. D. Moll, A. L. Gaeta, and G. Fibich, Phys. Rev. Lett. 90, 203902 (2003); A. L. Gaeta, Science 301, 54 (2003).

[12] L. T. Vuong et al., Phys. Rev. Lett. 96, 133901 (2006).

[13] G. Chang et al., Phys. Rev. E 72, 016609 (2005).

[14] L. Wu et al., Opt. Express 16, 6352 (2008).

[15] S. Trillo and W. Torruellas, Spatial Solitons (Springer, Berlin, 2001).

[16] A. V. Buryak et al., Phys. Rep. 370, 63 (2002).

[17] A. Desyatnikov, A. Maimistov, and B. Malomed, Phys. Rev. E 61, 3107 (2000).

[18] M. Belić et al., Phys. Rev. Lett. 101, 123904 (2008). 